\title{
SELF-CONTRADICTION AND CONTRADICTION BETWEEN TWO ATANASSOV'S INTUITIONISTIC FUZZY SETS*
}

\author{
SUSANA CUBILLO, CARMEN TORRES and ELENA CASTIÑEIRA \\ Department of Applied Mathematics, Technical University of Madrid, 28660 Boadilla del Monte \\ Madrid, Spain
}

\begin{abstract}
The paper focuses on the study of the contradiction between two Atanassov's intuitionistic fuzzy sets. First, taking into account some characterizations obtained in previous papers, some functions are defined in order to measure the degrees of contradiction. Besides the principal properties of these measures are pointed out. Finally, some results relating self-contradiction and contradiction between two Atanassov's intuitionistic fuzzy sets are achieved.

Keywords: Atanassov's Intuitionistic fuzzy sets, intuitionistic fuzzy generators and fuzzy negations, degrees of contradiction.
\end{abstract}

\section{Introduction}

In many of the applications in the fuzzy inference systems, contradictory outputs could be obtained. In order to prevent this kind of problem it is important to raise the study of self-contradiction. Besides, the study of contradiction between sets seems suitable because it is also necessary to avoid, in the inference processes, obtaining contradictory outputs from inputs. The study of the contradiction in the Fuzzy Logic was initiated by Trillas Later, focused on the problem of determining to what extent a fuzzy set is contradictory, giving some functions for this purpose.

Also, the contradiction is tackled in the framework of the Atanassov's intuitionistic fuzzy sets (A-IFSs) introducing the concepts of self-contradictory A-IFS and contradiction between two A-IFSs. In the degrees in which an A-IFS is selfcontradictory are studied providing some functions to measure them.

The present paper focuses on two mains subjects. The first one (sections 2 and 3 ) is the study of degrees of contradiction between two A-IFSs, meanwhile the second 
one (section 4) is to relate contradiction between two A-IFSs and self-contradiction.

Let us remember that:

Definition 1. ${ }^{1}$ ) An Atanassov's intuitionistic fuzzy set (A-IFS) $A$, in the universe $X \neq \emptyset$, is a set given as $A=\left\{\left(x, \mu_{A}(x), \nu_{A}(x)\right): x \in X\right\}$, where $\mu_{A}: X \rightarrow[0,1]$, $\nu_{A}: X \rightarrow[0,1]$ are called membership and non-membership functions, respectively, and such that $\mu_{A}(x)+\nu_{A}(x) \leq 1$ holds for all $x \in X$.

Furthermore, an A-IFS could be considered as an $L$-fuzzy set as defined by Goguen ${ }^{9}$ being, in this case, $L=\left\{\left(\alpha_{1}, \alpha_{2}\right) \in[0,1]^{2}: \alpha_{1}+\alpha_{2} \leq 1\right\}$, with the partial order $\leq_{L}$ defined as follows: given $\boldsymbol{\alpha}=\left(\alpha_{1}, \alpha_{2}\right), \boldsymbol{\beta}=\left(\beta_{1}, \beta_{2}\right) \in L$,

$$
\boldsymbol{\alpha} \leq_{L} \boldsymbol{\beta} \Longleftrightarrow \alpha_{1} \leq \beta_{1} \& \alpha_{2} \geq \beta_{2}
$$

$\left(L, \leq_{L}\right)$ is a complete lattice with smallest element, $\mathbf{0}_{\mathbf{L}}=(0,1)$, and greatest element, $\mathbf{1}_{\mathbf{L}}=(1,0)$.

Thus, an A-IFS $A$ is an $L$-fuzzy set whose $L$-membership function $\chi^{A} \in L^{X}=$ $\{\chi: X \rightarrow L\}$ is defined for each $x \in X$ as $\chi^{A}(x)=\left(\mu_{A}(x), \nu_{A}(x)\right)$, and let us denote $\chi^{A}(X)=\left\{\chi^{A}(x): x \in X\right\}$ the image of $X$ under $\chi^{A}$, that we also call range of $A$. Let us denote the set of all intuitionistic (or Atanassov's) fuzzy sets on $X$ as $\mathcal{A I F}(X)$.

Also, let us recall that a decreasing function $\mathcal{N}: L \rightarrow L$ is an intuitionistic fuzzy negation (IFN) if $\mathcal{N}\left(\mathbf{0}_{\mathrm{L}}\right)=\mathbf{1}_{\mathrm{L}}$ and $\mathcal{N}\left(\mathbf{1}_{\mathrm{L}}\right)=\mathbf{0}_{\mathrm{L}}$ hold. Moreover, $\mathcal{N}$ is a strong IFN if the equality $\mathcal{N}(\mathcal{N}(\alpha))=\alpha$ holds for all $\alpha \in L$. Bustince et al. in ${ }^{2}$ introduced the intuitionistic fuzzy generators, that can be used to build intuitionistic fuzzy negations, and Deschrijver et al. in ${ }^{8}$ proved that any strong IFN $\mathcal{N}$ is characterized by a strong negation $N:[0,1] \rightarrow[0,1]$ by means of the formula $\mathcal{N}\left(\alpha_{1}, \alpha_{2}\right)=$ $\left(N\left(1-\alpha_{2}\right), 1-N\left(\alpha_{1}\right)\right)$, for all $\left(\alpha_{1}, \alpha_{2}\right) \in L$. Furthermore, let us remember that Trillas in ${ }^{10}$ showed that $N$ is a strong negation if and only if there exists an order automorphism in the unit interval $g(g:[0,1] \rightarrow[0,1]$ a bijective and strictly increasing function), such that $N(\alpha)=g^{-1}(1-g(\alpha))$, for all $\alpha \in[0,1]$, and it will be said that $g$ is the generator of negation $N$.

Thus a strong IFN $\mathcal{N}$ is also determined or generated by an order automorphism $g$ in $[0,1]$. Sometimes we will denote $\mathcal{N}=\mathcal{N}_{g}$ in reference to this fact, and we will say that $\mathcal{N}_{g}$ is given by $g$.

\section{Degrees of $\mathcal{N}$-Contradiction between two A-IFSs}

The study of contradiction between two sets is interesting because, in the inference process, it is desirable to avoid new results being incoherent with the previous information. In this section we deal with degrees of contradiction between two AIFSs when a strong IFN, $\mathcal{N}$, is considered; that is, the degrees of $\mathcal{N}$-contradiction. For this, we introduce some functions and study some of their properties.

In ${ }^{7}$, it was established that, given $A, B \in \mathcal{A I \mathcal { F }}(X)$ the A-IFSs determined by $\chi^{A}=\left(\mu_{A}, \nu_{A}\right) \in L^{X}$, and $\chi^{B}=\left(\mu_{B}, \nu_{B}\right) \in L^{X}$, and a strong IFN, $\mathcal{N}$, associated 
with the strong negation $N$, it will be said that $A$ and $B$ are $\mathcal{N}$-contradictory if $\chi^{A} \leq_{L} \mathcal{N} \circ \chi^{B}$, that is, for all $x \in X, \chi^{A}(x) \leq_{L}\left(\mathcal{N} \circ \chi^{B}\right)(x)$.

Furthermore, some characterizations were reached in the same paper:

(i) $A$ and $B$ are $\mathcal{N}$-contradictory if and only if the following inequalities hold

$$
N\left(\mu_{A}(x)\right)+\nu_{B}(x) \geq 1 \text { and } N\left(\mu_{B}(x)\right)+\nu_{A}(x) \geq 1, \quad \forall x \in X
$$

(ii) $A$ and $B$ are $\mathcal{N}$-contradictory if and only if

$$
g\left(\mu_{A}(x)\right)+g\left(1-\nu_{B}(x)\right) \leq 1 \text { and } g\left(\mu_{B}(x)\right)+g\left(1-\nu_{A}(x)\right) \leq 1, \quad \forall x \in X
$$

where $g$ is the generator of the negation $N$ associated to $\mathcal{N}$.

From these characterizations, the following result is reached:

Lemma 1. Given $A, B \in \mathcal{A} \mathcal{I} \mathcal{F}(X)$ with membership/non-membership function (L-membership function) $\chi^{A}=\left(\mu_{A}, \nu_{A}\right) \in L^{X}$ and $\chi^{B}=\left(\mu_{B}, \nu_{B}\right) \in L^{X}$ respectively, and given $\mathcal{N}$ the strong IFN generated by $g$, if $A, B$ are $\mathcal{N}$-contradictory then $g\left(\mu_{A}(x)\right)+g\left(\mu_{B}(x)\right) \leq 1$. Besides, if $g\left(1-\nu_{A}(x)\right)+g\left(1-\nu_{B}(x)\right) \leq 1$ then $A, B$ are $\mathcal{N}$-contradictory.

Proof. As $A, B$ are A-IFSs, $\mu_{A}(x)+\nu_{A}(x) \leq 1$ and $\mu_{B}(x)+\nu_{B}(x) \leq 1$ and so $g\left(\mu_{A}(x)\right) \leq g\left(1-\nu_{A}(x)\right)$ and $g\left(\mu_{B}(x)\right) \leq g\left(1-\nu_{B}(x)\right)$, for all $g$ order automorphism.

$A, B$ are $\mathcal{N}$-contradictory if and only if $g\left(\mu_{A}(x)\right)+g\left(1-\nu_{B}(x)\right) \leq 1$ and $g\left(\mu_{B}(x)\right)+g\left(1-\nu_{A}(x)\right) \leq 1$, being $g$ the order automorphism associated to $\mathcal{N}$. Then, $g\left(\mu_{A}(x)\right)+g\left(\mu_{B}(x)\right) \leq g\left(1-\nu_{A}(x)\right)+g\left(\mu_{B}(x)\right) \leq 1$

Now, if $g\left(1-\nu_{A}(x)\right)+g\left(1-\nu_{B}(x)\right) \leq 1$ then $g\left(\mu_{A}(x)\right)+g\left(1-\nu_{B}(x)\right) \leq g(1-$ $\left.\nu_{A}(x)\right)+g\left(1-\nu_{B}(x)\right) \leq 1$ and $g\left(\mu_{B}(x)\right)+g\left(1-\nu_{A}(x)\right) \leq g\left(1-\nu_{B}(x)\right)+g\left(1-\nu_{A}(x)\right) \leq$ 1 ; thereby $A, B$ are $\mathcal{N}$-contradictory.

Remark 1. Let us note that a fuzzy set could be considered as an A-IFS $A$ where $\nu_{A}(x)=1-\mu_{A}(x)$ for all $x \in X$. So, if we have $A$ and $B$ two fuzzy sets, and the strong IFN $\mathcal{N}$ is determined by the strong fuzzy negation $N_{g}$, then $A, B$ are $\mathcal{N}$ contradictory if and only if $g\left(\mu_{A}(x)\right)+g\left(\mu_{B}(x)\right) \leq 1$ for all $x \in X$. This condition, in the framework of the fuzzy sets, means that $A, B$ are $N_{g}$-contradictory. So, the contradiction in fuzzy sets is a particular case of the contradiction give here.

Let us observe that the curve $N\left(\alpha_{1}\right)+\alpha_{2}=1$ (that, of course, is coincidental with $\left.g\left(\alpha_{1}\right)+g\left(1-\alpha_{2}\right)=1\right)$, points out the limit of the contradiction region.

Given $A, B \in \mathcal{A} \mathcal{I} \mathcal{F}(X)$ determined by $\chi^{A}=\left(\mu_{A}, \nu_{A}\right), \chi^{B}=\left(\mu_{B}, \nu_{B}\right) \in L^{X}$, we will denote $\chi^{A B}=\left(\mu_{A}, \nu_{B}\right)$, and $\chi^{B A}=\left(\mu_{B}, \nu_{A}\right) \cdot \chi^{A B}$ and $\chi^{B A} \in([0,1] \times[0,1])^{X}$ and are not, in general, A-IFSs because they could take values out of $L$. Besides if we consider the set $\mathcal{R}_{\mathcal{N}}=\left\{\left(\alpha_{1}, \alpha_{2}\right) \in[0,1]^{2}: N\left(\alpha_{1}\right)+\alpha_{2} \geq 1\right\}, A$ and $B$ are $\mathcal{N}$-contradictory if and only if

$$
\chi^{A B}(X) \subset \mathcal{R}_{\mathcal{N}} \text { and } \chi^{B A}(X) \subset \mathcal{R}_{\mathcal{N}}
$$


and the $\mathcal{N}$-boundary curve delimiting the $\mathcal{N}$-contradiction region, $N\left(\alpha_{1}\right)+\alpha_{2}=1$, is the same that the curve given for delimiting the self- $\mathcal{N}$-contradiction region, but now $\alpha_{1}$ takes its values in $[0,1]$, and not only in $\left[0, \alpha_{N}\right]$, where $\alpha_{N}$ is the equilibrium point of the fuzzy negation $N$, that is the only value in $(0,1)$ satisfying $N\left(\alpha_{N}\right)=\alpha_{N}$ (then $\alpha_{N}=g^{-1}\left(\frac{1}{2}\right)$ being $g$ the generator of $N$ ). The curve $N\left(\alpha_{1}\right)+\alpha_{2}=1$ satisfies the following properties (see figure 1):

(1) $\alpha_{2}=1-N\left(\alpha_{1}\right)$ is an increasing function of the variable $\alpha_{1}$.

(2) Its range contains the points $(0,0),(1,1)$ and $\left(\alpha_{N}, 1-\alpha_{N}\right)$.

(3) It is symmetrical with respect to the line $\alpha_{1}+\alpha_{2}=1$.

(4) The intersection of $N\left(\alpha_{1}\right)+\alpha_{2}=1$ and $\alpha_{1}+\alpha_{2}=1$ is the point $\left(\alpha_{N}, 1-\alpha_{N}\right)$.

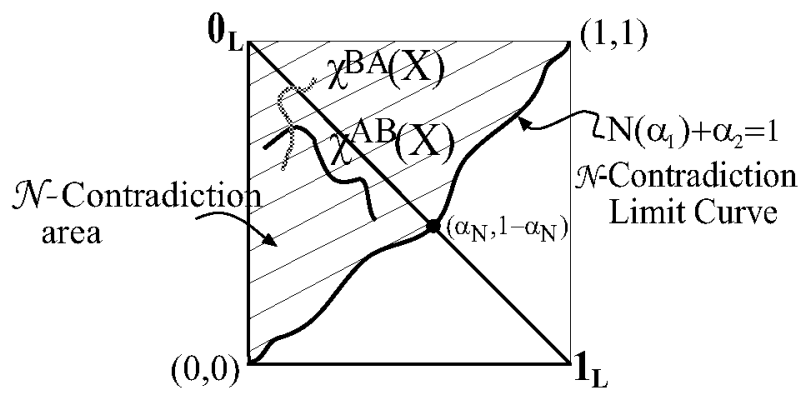

Fig. 1. Region of $\mathcal{N}$-contradiction.

All that presented in this section suggests us to determine some ways to define the $\mathcal{N}$-contradiction degrees between two A-IFSs.

Definition 2. Given $A, B \in \mathcal{A} \mathcal{I} \mathcal{F}(X)$ determined by $\chi^{A}=\left(\mu_{A}, \nu_{A}\right), \chi^{B}=$ $\left(\mu_{B}, \nu_{B}\right) \in L^{X}$, respectively, and $\mathcal{N}$ the strong IFN generated by $g$, we define the $\mathcal{C}_{i}^{\mathcal{N}}$-contradiction degree between $A$ and $B$, for $i=1,2,3$, as follows:

(i) $\mathcal{C}_{1}^{\mathcal{N}}(A, B)=\operatorname{Max}\left(0, \operatorname{Min}\left(\operatorname{Inf}_{x \in X}\left(N_{g}\left(\mu_{A}(x)\right)+\nu_{B}(x)-1\right), \operatorname{Inf}_{x \in X}\left(N_{g}\left(\mu_{B}(x)\right)+\nu_{A}(x)-\right.\right.\right.$ 1)))

(ii) $\mathcal{C}_{2}^{\mathcal{N}}(A, B)=\operatorname{Max}\left(0, \operatorname{Min}\left(1-\operatorname{Sup}_{x \in X}\left(g\left(\mu_{A}(x)\right)+g\left(1-\nu_{B}(x)\right)\right), 1-\operatorname{Sup}_{x \in X}\left(g\left(\mu_{B}(x)\right)+\right.\right.\right.$ $\left.\left.\left.g\left(1-\nu_{A}(x)\right)\right)\right)\right)$

(iii) Let $\mathcal{R}_{\mathcal{N}}^{*}=\left\{\alpha=\left(\alpha_{1}, \alpha_{2}\right) \in[0,1]^{2} ; N_{g}\left(\alpha_{1}\right)+\alpha_{2} \leq 1\right\}$, then

$$
\mathcal{C}_{3}^{\mathcal{N}}(A, B)=\frac{\operatorname{Min}\left(d\left(\chi^{A B}(X), \mathcal{R}_{\mathcal{N}}^{*}\right), d\left(\chi^{B A}(X), \mathcal{R}_{\mathcal{N}}^{*}\right)\right)}{d\left(\mathbf{0}_{\mathbf{L}}, \mathcal{R}_{\mathcal{N}}^{*}\right)}
$$

where $d$ is the euclidean distance.

Let us note that $\mathcal{C}_{1}^{\mathcal{N}}$ is suggested by the first characterization of $\mathcal{N}$-contradictory sets; $\mathcal{C}_{2}^{\mathcal{N}}$ by the second one, and $\mathcal{C}_{3}^{\mathcal{N}}$ by the distance between the range of the sets 
$\chi^{A B}, \chi^{B A}$ and the region of non-contradiction.

Example 1. Let $A, B$ be the A-IFSs given by the $L$-membership functions $\chi^{A}(x)=$ $\left(\frac{x}{4}, 1-\frac{x}{2}\right)$ and $\chi^{B}(x)=\left(\frac{x}{3}, 1-\frac{x}{2}\right)$, respectively, where $x \in X=[0,1]$ (see figure 2); it is easy to prove they are well defined.

Let us calculate the contradiction degrees when the standard negation is considered, that is, $\mathcal{N}_{s}\left(\alpha_{1}, \alpha_{2}\right)=\left(\alpha_{2}, \alpha_{1}\right)$ (associated with the fuzzy negation $N_{s}(x)=$ $1-x$, and $g=i d)$ :

(i) $\mathcal{C}_{1}^{\mathcal{N}_{s}}(A, B)=\operatorname{Max}\left(0, \operatorname{Min}\left(\operatorname{Inf}_{x \in X}\left(1-\frac{x}{4}+1-\frac{x}{2}-1\right), \operatorname{Inf}_{x \in X}\left(1-\frac{x}{3}+1-\frac{x}{2}-1\right)\right)\right)=$ $\operatorname{Max}\left(0, \operatorname{Min}\left(\frac{1}{4}, \frac{1}{6}\right)\right)=\frac{1}{6}$

(ii) $\mathcal{C}_{2}^{\mathcal{N}_{s}}(A, B)=\operatorname{Max}\left(0, \operatorname{Min}\left(1-\operatorname{Sup}_{x \in X}\left(\frac{x}{4}+\frac{x}{2}\right), 1-\operatorname{Sup}_{x \in X}\left(\frac{x}{3}+\frac{x}{2}\right)\right)\right)=$ $\operatorname{Max}\left(0, \operatorname{Min}\left(\frac{1}{4}, \frac{1}{6}\right)\right)=\frac{1}{6}$

(iii) The infimum, when $x \in X$, of the distances from $\left(\mu_{A}(x), \nu_{B}(x)\right)=\left(\frac{x}{4}, 1-\frac{x}{2}\right)$ to the area $\mathcal{R}_{\mathcal{N}}^{*}=\left\{\left(\alpha_{1}, \alpha_{2}\right) ; \alpha_{1} \geq \alpha_{2}\right\}$ is the value $\frac{\sqrt{2}}{8}$. Meanwhile the infimum, when $x \in X$, of the distances from $\left(\mu_{B}(x), \nu_{A}(x)\right)=\left(\frac{x}{3}, 1-\frac{x}{2}\right)$ to $\mathcal{R}_{\mathcal{N}}^{*}$ is $\frac{\sqrt{2}}{12}$; then $\mathcal{C}_{3}^{\mathcal{N}_{s}}(A, B)=\frac{\sqrt{2} / 12}{d\left(\mathbf{0}_{\mathrm{L}}, \mathcal{R}_{\mathcal{N}}^{*}\right)}=\frac{1}{6}$

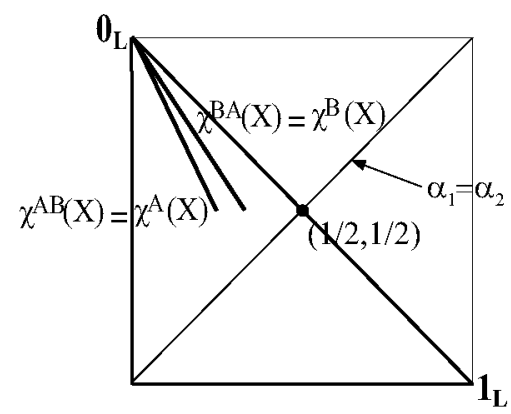

Fig. 2. Example of $\mathcal{N}_{s}$-contradictory sets.

The above example shows a situation in which the three functions $\mathcal{C}_{i}^{\mathcal{N}}, \mathrm{i}=1,2,3$, coincide; in fact we have the following more general result:

Proposition 1. Given the standard IFN $\mathcal{N}_{s}$, the equalities $\mathcal{C}_{1}^{\mathcal{N}_{s}}(A, B)=$ $\mathcal{C}_{2}^{\mathcal{N}_{s}}(A, B)=\mathcal{C}_{3}^{\mathcal{N}_{s}}(A, B)$ hold for all $A, B \in \mathcal{A} \mathcal{I} \mathcal{F}(X)$.

Now let us see some examples in which the contradiction degrees are different.

Example 2. With the two A-IFSs given in the example 1, let us now consider the negation $N_{g}(x)=\sqrt{1-x^{2}}$, whose generator is $g(x)=x^{2}$ : 
(i) $\mathcal{C}_{1}^{\mathcal{N}}(A, B)=\operatorname{Max}\left(0, \operatorname{Min}\left(\operatorname{Inf}_{x \in X}\left(\sqrt{1-\left(\frac{x}{4}\right)^{2}}-\frac{x}{2}\right), \operatorname{Inf}_{x \in X}\left(\sqrt{1-\left(\frac{x}{3}\right)^{2}}-\frac{x}{2}\right)\right)\right)=$ $\operatorname{Max}\left(0, \operatorname{Min}\left(\sqrt{\frac{15}{16}}-\frac{1}{2}, \sqrt{\frac{8}{9}}-\frac{1}{2}\right)\right)=\frac{\sqrt{8}}{3}-\frac{1}{2}$

(ii) $\mathcal{C}_{2}^{\mathcal{N}}(A, B)=\operatorname{Max}\left(0, \operatorname{Min}\left(1-\operatorname{Sup}_{x \in X}\left(\left(\frac{x}{4}\right)^{2}+\left(\frac{x}{2}\right)^{2}\right), 1-\operatorname{Sup}_{x \in X}\left(\left(\frac{x}{3}\right)^{2}+\left(\frac{x}{2}\right)^{2}\right)\right)\right)=$ $\operatorname{Max}\left(0, \operatorname{Min}\left(1-\frac{5}{16}, 1-\frac{13}{36}\right)\right)=\frac{23}{36}$

Example 3. Let $A, B$ be the A-IFSs given by the $L$-membership functions $\chi^{A}(x)=$ $\left(\frac{1}{4}-\frac{x}{6}, \frac{1}{4}+\frac{x}{2}\right)$ and $\chi^{B}(x)=\left(\frac{1-x}{3}, \frac{1+x}{2}\right)$, where $x \in X=[0,1]$ (see figure 3 ); it can be proved they are well defined.

If $\mathcal{N}$ is the IFN associated with the negation $N_{g}(x)=\sqrt{1-x^{2}}$, whose generator is $g(x)=x^{2}$, the contradiction degrees between $A$ an $B$ are:

(i) $\mathcal{C}_{1}^{\mathcal{N}}(A, B)=\operatorname{Max}\left(0, \operatorname{Min}\left(\operatorname{Inf}_{x \in X}\left(\sqrt{1-\left(\frac{1}{4}-\frac{x}{6}\right)^{2}}+\frac{1+x}{2}-1\right), \operatorname{Inf}_{x \in X}\left(\sqrt{1-\left(\frac{1-x}{3}\right)^{2}}+\right.\right.\right.$ $\left.\left.\left.\frac{1}{4}+\frac{x}{2}-1\right)\right)\right)=\operatorname{Max}\left(0, \operatorname{Min}\left(\sqrt{\frac{15}{16}}-\frac{1}{2}, \sqrt{\frac{8}{9}}-\frac{3}{4}\right)\right)=\frac{2 \sqrt{2}}{3}-\frac{3}{4}$

(ii) $\mathcal{C}_{2}^{\mathcal{N}}(A, B)=\operatorname{Max}\left(0, \operatorname{Min}\left(1-\operatorname{Sup}_{x \in X}\left(\left(\frac{1}{4}-\frac{x}{6}\right)^{2}+\left(1-\frac{1+x}{2}\right)^{2}\right), 1-\operatorname{Sup}_{x \in X}\left(\left(\frac{1-x}{3}\right)^{2}+(1-\right.\right.\right.$ $\left.\left.\left.\left.\left.\frac{1}{4}-\frac{x}{2}\right)^{2}\right)\right)\right)=\operatorname{Max}\left(0, \operatorname{Min}\left(1-\left(\frac{1}{4^{2}}+\frac{1}{2^{2}}\right)\right), 1-\left(\frac{1}{3^{2}}+\left(\frac{3}{4}\right)^{2}\right)\right)\right)=1-\left(\frac{1}{3^{2}}+\left(\frac{3}{4}\right)^{2}\right)$

(iii) As the free of contradiction region is $\mathcal{R}_{\mathcal{N}}^{*}=\left\{\left(\alpha_{1}, \alpha_{2}\right) \in[0,1]^{2}: \alpha_{1}^{2}+\left(1-\alpha_{2}\right)^{2} \geq\right.$ $1\}$, then

$$
\begin{aligned}
\mathcal{C}_{3}^{\mathcal{N}}(A, B) & =\operatorname{Min}\left(d\left(\chi^{A B}(X), \mathcal{R}_{\mathcal{N}}^{*}\right), d\left(\chi^{B A}(X), \mathcal{R}_{\mathcal{N}}^{*}\right)\right) \\
& =\operatorname{Min}\left(d\left(\left(\frac{1}{4}, \frac{1}{2}\right), \mathcal{R}_{\mathcal{N}}^{*}\right)\right), d\left(\left(\frac{1}{3}, \frac{1}{4}\right), \mathcal{R}_{\mathcal{N}}^{*}\right) \\
& =1-d((1 / 3,1 / 4),(0,1)) \\
& =1-\sqrt{1 / 3^{2}+(3 / 4)^{2}}
\end{aligned}
$$
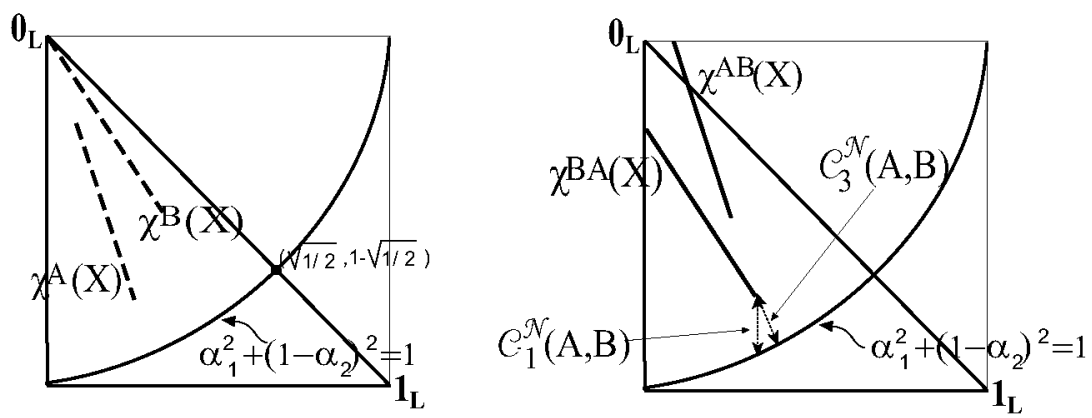

Fig. 3. $A$ and $B$ with different contradiction degrees.

Despite no connection among $\mathcal{C}_{i}^{\mathcal{N}}$ for $i=1,2,3$ holds in general, there is a relation between functions $\mathcal{C}_{2}^{\mathcal{N}}$ and $\mathcal{C}_{3}^{\mathcal{N}}$ when we deal with a specific negation, as the following result shows. 
Proposition 2. Given $\mathcal{N}$ the strong IFN generated by the automorphism $g(x)=x^{2}$, then $\mathcal{C}_{2}^{\mathcal{N}}(A, B)=1-\left(1-\mathcal{C}_{3}^{\mathcal{N}}(A, B)\right)^{2}$ is satisfied for all $A, B \in \mathcal{A I \mathcal { F }}(X)$

The following properties can be proved:

Theorem 1. For $i=1,2,3$, the function $\mathcal{C}_{i}^{\mathcal{N}}: \mathcal{A} \mathcal{I} \mathcal{F}(X) \times \mathcal{A I F}(X) \rightarrow[0,1]$, given for each $A, B \in \mathcal{A I \mathcal { F }}(X)$ as in the above definition, satisfies:

(i) $\mathcal{C}_{i}^{\mathcal{N}}$ is a symmetrical function: $\mathcal{C}_{i}^{\mathcal{N}}(A, B)=\mathcal{C}_{i}^{\mathcal{N}}(B, A)$ for all $A, B \in \mathcal{A I F}(X)$.

(ii) If $0_{\mathrm{L}}$ denotes the A-IFS such that $\chi^{0_{\mathrm{L}}}(x)=0_{\mathrm{L}}$ for all $x \in X$, then $\mathcal{C}_{i}^{\mathcal{N}}\left(0_{\mathrm{L}}, \mathbf{0}_{\mathbf{L}}\right)=1$.

(iii) If $\operatorname{Inf}_{x \in X} \nu_{A}(x)=0$ or $\operatorname{Inf}_{x \in X} \nu_{B}(x)=0$ then $\mathcal{C}_{i}^{\mathcal{N}}(A, B)=0$.

(iv) $\mathcal{C}_{i}^{\mathcal{N}}$ is anti-monotonic at both variables, with respect to the orders $\leq_{L}$ in $L$ and the usual one in $\mathbb{R}$ : If $A, B, C \in \mathcal{A T \mathcal { F }}(X)$ with $\chi^{A} \leq_{L} \chi^{B}$ (that is, $\chi^{A}(x) \leq_{L}$ $\chi^{B}(x)$ for all $\left.x \in X\right)$, then $\mathcal{C}_{i}^{\mathcal{N}}(B, C) \leq \mathcal{C}_{i}^{\mathcal{N}}(A, C)$.

\section{Proof.}

(i) $\mathcal{C}_{i}^{\mathcal{N}}(A, B)=\mathcal{C}_{i}^{\mathcal{N}}(B, A)$ for $i=1,2,3$ trivially for the above definitions.

(ii) $\mathcal{C}_{1}^{\mathcal{N}}\left(\mathbf{0}_{\mathbf{L}}, \mathbf{0}_{\mathbf{L}}\right)=\operatorname{Max}\left(\mathbf{0}, \operatorname{Min}\left(\operatorname{Inf}_{\boldsymbol{x} \in X}\left(N_{g}(\mathbf{0})+1-1\right), \operatorname{Inf}_{\boldsymbol{x} \in X}\left(N_{g}(0)+1-1\right)\right)\right)=1$

$\mathcal{C}_{2}^{\mathcal{N}}\left(\mathbf{0}_{\mathbf{L}}, \mathbf{0}_{\mathbf{L}}\right)=\operatorname{Max}\left(0, \operatorname{Min}\left(1-\operatorname{Sup}_{x \in X}(g(0)+g(1-1)), 1-\operatorname{Sup}_{x \in X}(g(0)+g(1-1))\right)\right)=1$

$\mathcal{C}_{3}^{\mathcal{N}}\left(\mathbf{0}_{\mathbf{L}}, \mathbf{0}_{\mathbf{L}}\right)=\frac{\operatorname{Min}\left(d\left(\mathbf{0}_{\mathbf{L}}, \mathcal{R}_{\mathcal{N}}^{*}\right), d\left(\mathbf{0}_{\mathbf{L}}, \mathcal{R}_{\mathcal{N}}^{*}\right)\right)}{d\left(\mathbf{0}_{\mathbf{L}}, \mathcal{R}_{\mathcal{N}}^{*}\right)}=1$

(iii) Let $A, B \in \mathcal{A} \mathcal{I F}(X)$ be two sets such that $\operatorname{Inf}_{x \in X} \nu_{A}(x)=0$ or $\operatorname{Inf}_{x \in X} \nu_{B}(x)=$ 0 . Since $N_{g}\left(\mu_{A}(x)\right) \leq 1$ and $N_{g}\left(\mu_{B}(x)\right) \leq 1$ for all $x \in X$, being $N_{g}$ the strong fuzzy negation associated with the strong $\operatorname{IFN} \mathcal{N}$, then $\operatorname{Inf}_{x \in X}\left(N_{g}\left(\mu_{B}(x)\right)+\right.$ $\left.\nu_{A}(x)-1\right) \leq \operatorname{Inf}_{x \in X}\left(\nu_{A}(x)\right)=0$ or $\operatorname{Inf}_{x \in X}\left(N_{g}\left(\mu_{A}(x)\right)+\nu_{B}(x)-1\right) \leq \operatorname{Inf}_{x \in X}\left(\nu_{B}(x)\right)=0$. Therefore, $\mathcal{C}_{1}^{\mathcal{N}}(A, B)=0$.

Now, if $g$ is the generator of negation $N_{g}$ associated to $\mathcal{N}$, then

$$
\operatorname{Sup}_{x \in X}\left(g\left(\mu_{B}(x)\right)+g\left(1-\nu_{A}(x)\right)\right) \geq \operatorname{Sup}_{x \in X}\left(g\left(1-\nu_{A}(x)\right)\right)=g\left(1-\operatorname{Inf}_{x \in X} \nu_{A}(x)\right)=1
$$

or

$\operatorname{Sup}_{x \in X}\left(g\left(\mu_{A}(x)\right)+g\left(1-\nu_{B}(x)\right)\right) \geq \operatorname{Sup}_{x \in X}\left(g\left(1-\nu_{B}(x)\right)\right)=g\left(1-\operatorname{Inf}_{x \in X} \nu_{B}(x)\right)=1$

and so, $\mathcal{C}_{2}^{\mathcal{N}}(A, B)=0$.

Since $\operatorname{Inf}_{x \in X} \nu_{A}(x)=0$ or $\operatorname{Inf}_{x \in X} \nu_{B}(x)=0$ there exists some $\left(\alpha_{0}, 0\right)$ belonging to the closure of $\chi^{B A}\left(\overline{\chi^{B A}}\right)$ or to that of $\chi^{A B}\left(\overline{\chi^{A B}}\right)$, then $\left(\alpha_{0}, 0\right) \in \mathcal{R}_{\mathcal{N}}^{*} \cap \overline{\chi^{B A}}$ or $\left(\alpha_{0}, 0\right) \in \mathcal{R}_{\mathcal{N}}^{*} \cap \overline{\chi^{A B}}$, consequently, it holds that $d\left(\chi^{B A}(X), \mathcal{R}_{\mathcal{N}}^{*}\right)=0$ or $d\left(\chi^{A B}(X), \mathcal{R}_{\mathcal{N}}^{*}\right)=0$. So, $\mathcal{C}_{3}^{\mathcal{N}}(A, B)=0$.

(iv) Let us see that the three functions are anti-monotonic. If $\chi^{A} \leq_{L} \chi^{B}$ then $\mu_{A}(x) \leq \mu_{B}(x)$ and $\nu_{A}(x) \geq \nu_{B}(x)$ for all $x \in X$. 
'I'herefore, $N_{g}\left(\mu_{B}(x)\right) \leq N_{g}\left(\mu_{A}(x)\right)$ and so $N_{g}\left(\mu_{B}(x)\right)+\nu_{C}(x)-1 \leq$ $N_{g}\left(\mu_{A}(x)\right)+\nu_{C}(x)-1$. Also, it is verified that $N_{g}\left(\mu_{C}(x)\right)+\nu_{B}(x)-1 \leq$ $N_{g}\left(\mu_{C}(x)\right)+\nu_{A}(x)-1$. Then, $\mathcal{C}_{1}^{\mathcal{N}}(B, C) \leq \mathcal{C}_{1}^{\mathcal{N}}(A, C)$.

In addition, as $g\left(\mu_{A}(x)\right) \leq g\left(\mu_{B}(x)\right)$ and $g\left(1-\nu_{A}(x)\right) \leq g\left(1-\nu_{B}(x)\right)$, it is $1-\operatorname{Sup}_{x \in X}\left(g\left(\mu_{B}(x)\right)+g\left(1-\nu_{C}(x)\right)\right) \leq 1-\operatorname{Sup}_{x \in X}\left(g\left(\mu_{A}(x)\right)+g\left(1-\nu_{C}(x)\right)\right)$ and $1-\operatorname{Sup}_{x \in X}\left(g\left(\mu_{C}(x)\right)+g\left(1-\nu_{B}(x)\right)\right) \leq 1-\operatorname{Sup}_{x \in X}\left(g\left(\mu_{C}(x)\right)+g\left(1-\nu_{A}(x)\right)\right)$. Then, $\mathcal{C}_{2}^{\mathcal{N}}(B, C) \leq \mathcal{C}_{2}^{\mathcal{N}}(A, C)$

To prove that all $x \in X$ satisfies $d\left(\chi^{A C}(x), \mathcal{R}_{\mathcal{N}}^{*}\right) \geq d\left(\chi^{B C}(x), \mathcal{R}_{\mathcal{N}}^{*}\right)$ and $d\left(\chi^{C A}(x), \mathcal{R}_{\mathcal{N}}^{*}\right) \geq d\left(\chi^{C B}(x), \mathcal{R}_{\mathcal{N}}^{*}\right)$ provided $\chi^{A} \leq_{L} \chi^{B}$, will suffice to check the anti-monotony of $\mathcal{C}_{3}^{\mathcal{N}}$. Let $\chi^{A C}(x)=\left(\mu_{A}(x), \nu_{C}(x)\right) \in[0,1]^{2}$ and $\chi^{B C}(x)=$ $\left(\mu_{B}(x), \nu_{C}(x)\right) \in[0,1]^{2}$. If $d\left(\chi^{A C}(x), \mathcal{R}_{\mathcal{N}}^{*}\right)=0$ then $\chi^{A C}(x) \in \overline{\mathcal{R}_{\mathcal{N}}^{*}}=\mathcal{R}_{\mathcal{N}}^{*}$ (since $\mathcal{R}_{\mathcal{N}}^{*}$ is a closed set), and so $N_{g}\left(\mu_{A}(x)\right)+\nu_{C}(x) \leq 1$. Since, $\chi^{A}(x) \leq_{L} \chi^{B}(x)$ then $N_{g}\left(\mu_{B}(x)\right)+\nu_{C}(x) \leq N_{g}\left(\mu_{A}(x)\right)+\nu_{C}(x) \leq 1$ and therefore $d\left(\chi^{B C}(x), \mathcal{R}_{\mathcal{N}}^{*}\right)=0$ and the inequality is satisfied. Now, let us suppose that $d\left(\chi^{A C}(x), \mathcal{R}_{\mathcal{N}}^{*}\right)>0$ then this distance is reached on the boundary of $\mathcal{R}_{\mathcal{N}}^{*}$, that is the $\mathcal{N}$-contradiction limit curve $B\left(\mathcal{R}_{\mathcal{N}}^{*}\right)=\left\{\left(\alpha_{1}, \alpha_{2}\right) \in[0,1]^{2}: N_{g}\left(\alpha_{1}\right)+\alpha_{2}=1\right\}$, since $\mathcal{R}_{\mathcal{N}}^{*}$ is a compact set. Let $\alpha^{*}=\left(\alpha_{1}^{*}, \alpha_{2}^{*}\right) \in B\left(\mathcal{R}_{\mathcal{N}}^{*}\right)$ be the point such that

$$
d\left(\chi^{A C}(x), \mathcal{R}_{\mathcal{N}}^{*}\right)=d\left(\chi^{A C}(x), \alpha^{*}\right)
$$

and we consider the following three intervals contained in the interval $\left[\chi^{A C}(x),\left(1, \nu_{C}(x)\right)\right]=\left\{\left(\alpha_{1}, \nu_{C}(x)\right) \in[0,1]^{2}: \mu_{A}(x) \leq \alpha_{1} \leq 1\right\}$ (see figure 4):

$$
\begin{gathered}
I_{1}=\mathcal{R}_{\mathcal{N}}^{*} \bigcap\left[\chi^{A C}(x),\left(1, \nu_{C}(x)\right)\right], \\
I_{2}=\left\{\left(\alpha_{1}, \nu_{C}(x)\right) \in[0,1]^{2}: \mu_{A}(x) \leq \alpha_{1} \leq \alpha_{1}^{*}\right\}
\end{gathered}
$$

and

$$
I_{3}=\left\{\left(\alpha_{1}, \nu_{C}(x)\right) \in[0,1]^{2}: \alpha_{1}^{*} \leq \alpha_{1} \leq N_{g}^{-1}\left(1-\nu_{C}(x)\right)\right\}
$$

The union of the three above intervals is the whole interval $\left[\chi^{A C}(x),\left(1, \nu_{C}(x)\right)\right]$ and we assume $\chi^{B C}(x) \in\left[\chi^{A C}(x),\left(1, \nu_{C}(x)\right)\right]$, since $\mu_{B}(x) \geq \mu_{A}(x)$.

If $\chi^{B C}(x) \in I_{1}$ then $d\left(\chi^{B C}(x), \mathcal{R}_{\mathcal{N}}^{*}\right)=0 \leq d\left(\chi^{A C}(x), \mathcal{R}_{\mathcal{N}}^{*}\right)$.

If $\chi^{B C}(x) \in I_{2}$ then $\mu_{A}(x) \leq \mu_{B}(x) \leq \alpha_{1}^{*}$ and so $0 \leq \alpha_{1}^{*}-\mu_{B}(x) \leq \alpha_{1}^{*}-\mu_{A}(x)$, therefore $\left(\alpha_{1}^{*}-\mu_{B}(x)\right)^{2} \leq\left(\alpha_{1}^{*}-\mu_{A}(x)\right)^{2}$ and

$$
\begin{gathered}
d\left(\chi^{B C}(x), \mathcal{R}_{\mathcal{N}}^{*}\right) \leq d\left(\chi^{B C}(x), \alpha^{*}\right)=\sqrt{\left(\alpha_{1}^{*}-\mu_{B}(x)\right)^{2}+\left(\alpha_{2}^{*}-\nu_{C}(x)\right)^{2}} \leq \\
\leq \sqrt{\left(\alpha_{1}^{*}-\mu_{A}(x)\right)^{2}+\left(\alpha_{2}^{*}-\nu_{C}(x)\right)^{2}}=d\left(\chi^{A C}(x), \alpha^{*}\right)
\end{gathered}
$$

If $\chi^{B C}(x) \in I_{3}$ then

$d\left(\chi^{B C}(x), \mathcal{R}_{\mathcal{N}}^{*}\right) \leq d\left(\chi^{B C}(x),\left(\mu_{B}(x), 1-N_{g}^{-1}\left(\mu_{B}(x)\right)\right)\right)=\nu_{C}(x)-\left(1-N_{g}^{-1}\left(\mu_{B}(x)\right)\right)$ 


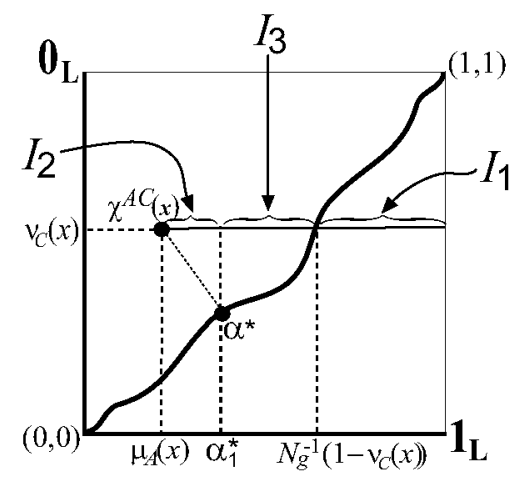

Fig. 4. The three intervals in $\left[\chi^{A C}(x),\left(1, \nu_{C}(x)\right)\right]$.

Moreover, $\alpha_{1}^{*} \leq \mu_{B}(x)$ implies $\alpha_{2}^{*}=1-N_{g}\left(\alpha_{1}^{*}\right) \leq 1-N_{g}\left(\mu_{B}(x)\right)$ and so

$\nu_{C}(x)-\left(1-N_{g}^{-1}\left(\mu_{B}(x)\right)\right) \leq \nu_{C}(x)-\alpha_{2}^{*} \leq \sqrt{\left(\mu_{A}(x)-\alpha_{1}^{*}\right)^{2}+\left(\nu_{C}(x)-\alpha_{2}^{*}\right)^{2}}=d\left(\chi^{A C}(x), \alpha^{*}\right)$

Now, Let $\chi^{C A}(x)=\left(\mu_{C}(x), \nu_{A}(x)\right) \in[0,1]^{2}$ and $\chi^{C B}(x)=\left(\mu_{C}(x), \nu_{B}(x)\right) \in$ $[0,1]^{2}$. If $d\left(\chi^{C A}(x), \mathcal{R}_{\mathcal{N}}^{*}\right)=0$ then $\chi^{C A}(x) \in \overline{\mathcal{R}_{\mathcal{N}}^{*}}=\mathcal{R}_{\mathcal{N}}^{*}$, and so $N_{g}\left(\mu_{C}(x)\right)+$ $\nu_{A}(x) \leq 1$. Since, $\chi^{A}(x) \leq{ }_{L} \chi^{B}(x)$ then $N_{g}\left(\mu_{C}(x)\right)+\nu_{B}(x) \leq N_{g}\left(\mu_{C}(x)\right)+$ $\nu_{A}(x) \leq 1$ and therefore $d\left(\chi^{C B}(x), \mathcal{R}_{\mathcal{N}}^{*}\right)=0$ and so $d\left(\chi^{C A}(x), \mathcal{R}_{\mathcal{N}}^{*}\right) \geq$ $d\left(\chi^{C B}(x), \mathcal{R}_{\mathcal{N}}^{*}\right)$. Now, let us suppose that $d\left(\chi^{C A}(x), \mathcal{R}_{\mathcal{N}}^{*}\right)>0$ and then exist a point $\alpha^{*}=\left(\alpha_{1}^{*}, \alpha_{2}^{*}\right) \in B\left(\mathcal{R}_{\mathcal{N}}^{*}\right)$ such that

$$
d\left(\chi^{C A}(x), \mathcal{R}_{\mathcal{N}}^{*}\right)=d\left(\chi^{C A}(x), \alpha^{*}\right)
$$

and we consider the following three intervals contained in the interval $\left[\left(\mu_{C}(x), 0\right), \chi^{C A}(x)\right]=\left\{\left(\mu_{C}(x), \alpha_{2}\right) \in[0,1]^{2}: 0 \leq \alpha_{2} \leq \nu_{A}(x)\right\}$ (see figure 5):

$$
\begin{gathered}
I_{1}^{\prime}=\mathcal{R}_{\mathcal{N}}^{*} \bigcap\left[\left(\mu_{C}(x), 0\right), \chi^{C A}(x)\right], \\
I_{2}^{\prime}=\left\{\left(\mu_{C}(x), \alpha_{2}\right) \in[0,1]^{2}: \alpha_{2}^{*} \leq \alpha_{2} \leq \nu_{A}(x)\right\}
\end{gathered}
$$

and

$$
I_{3}^{\prime}=\left\{\left(\mu_{C}(x), \alpha_{2}\right) \in[0,1]^{2}: 1-N_{g}\left(\mu_{C}(x)\right) \leq \alpha_{2} \leq \alpha_{2}^{*}\right\}
$$

The union of these three intervals is the whole interval $\left[\left(\mu_{C}(x), 0\right), \chi^{C A}(x)\right]$ and we assume $\chi^{C B}(x) \in\left[\left(\mu_{C}(x), 0\right), \chi^{C A}(x)\right]$, since $\nu_{B}(x) \leq \nu_{A}(x)$.

If $\chi^{C B}(x) \in I_{1}^{\prime}$ then $d\left(\chi^{C B}(x), \mathcal{R}_{\mathcal{N}}^{*}\right)=0 \leq d\left(\chi^{C A}(x), \mathcal{R}_{\mathcal{N}}^{*}\right)$.

If $\chi^{C B}(x) \in I_{2}^{\prime}$ then $\alpha_{2}^{*} \leq \nu_{B}(x) \leq \nu_{A}(x)$ and so $0 \leq \nu_{B}(x)-\alpha_{2}^{*} \leq \nu_{A}(x)-\alpha_{2}^{*}$, therefore $\left(\nu_{B}(x)-\alpha_{2}^{*}\right)^{2} \leq\left(\nu_{A}(x)-\alpha_{2}^{*}\right)^{2}$ and

$$
\begin{gathered}
d\left(\chi^{C B}(x), \mathcal{R}_{\mathcal{N}}^{*}\right) \leq d\left(\chi^{C B}(x), \alpha^{*}\right)=\sqrt{\left(\mu_{C}(x)-\alpha_{1}^{*}\right)^{2}+\left(\nu_{B}(x)-\alpha_{2}^{*}\right)^{2}} \leq \\
\leq \sqrt{\left(\mu_{C}(x)-\alpha_{1}^{*}\right)^{2}+\left(\nu_{A}(x)-\alpha_{2}^{*}\right)^{2}}=d\left(\chi^{C A}(x), \alpha^{*}\right)
\end{gathered}
$$




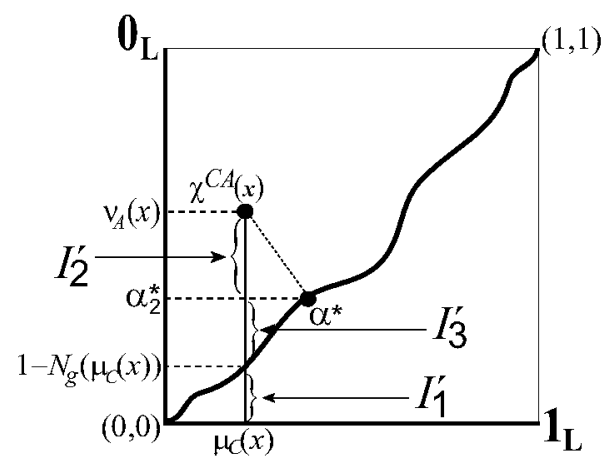

Fig. 5. The three intervals in $\left[\left(\mu_{C}(x), 0\right), \chi^{C A}(x)\right]$.

If $\chi^{C B}(x) \in I_{3}^{\prime}$ then $d\left(\chi^{C B}(x), \mathcal{R}_{\mathcal{N}}^{*}\right) \leq d\left(\chi^{C B}(x),\left(N_{g}^{-1}\left(1-\nu_{B}(x)\right), \nu_{B}(x)\right)\right)=$
$N_{g}^{-1}\left(1-\nu_{B}(x)\right)-\mu_{C}(x)$. Moreover, $\nu_{B}(x) \leq \alpha_{2}^{*}$ implies $N_{g}^{-1}\left(1-\nu_{B}(x)\right) \leq$
$N_{g}^{-1}\left(1-\alpha_{2}^{*}\right)=\alpha_{1}^{*}$ and so $N_{g}^{-1}\left(1-\nu_{B}(x)\right)-\mu_{C}(x) \leq \alpha_{1}^{*}-\mu_{C}(x) \leq$
$\sqrt{\left(\alpha_{1}^{*}-\mu_{C}(x)\right)^{2}+\left(\alpha_{2}^{*}-\nu_{A}(x)\right)^{2}}=d\left(\chi^{C A}(x), \alpha^{*}\right)$.

\section{Degrees of Contradiction between two A-IFSs}

Until now, the work has focused on contradiction depending on a given strong negation; but it is possible to measure the contradiction without depending on a specific IFN, as it was done in the case of a unique A-IFS. To this purpose, we remember a definition given, and we give some previous results will allow us to define several contradiction degrees.

Given $A, B \in \mathcal{A} \mathcal{I} \mathcal{F}(X)$, it will be said that $A$ and $B$ are contradictory if they are $\mathcal{N}$-contradictory regarding some strong $\operatorname{IFN} \mathcal{N}$.

Lemma 2. Given $A, B \in \mathcal{A} \mathcal{I} \mathcal{F}(X)$ with membership functions $\mu_{A}, \mu_{B} \in[0,1]^{X}$, respectively, if $A, B$ are contradictory then $\mu_{A}(x)+\mu_{B}(x)<2$ for all $x \in X$.

Proof. If $A, B$ are contradictory then $g\left(\mu_{A}(x)\right)+g\left(\mu_{B}(x)\right) \leq 1$ for some $g$ order automorphism (by lemma 1).

Now, we suppose $\operatorname{Sup}_{x \in X}\left(\mu_{A}(x)+\mu_{B}(x)\right)=2$ then $\mu_{A}(x)+\mu_{B}(x)>1+g^{-1}\left(\frac{1}{2}\right)$ for some $x \in X$, thereby $\mu_{A}(x)>g^{-1}\left(\frac{1}{2}\right)$ and $\mu_{B}(x)>g^{-1}\left(\frac{1}{2}\right)$ and consequently $g\left(\mu_{A}(x)\right)+g\left(\mu_{B}(x)\right)>1$, which is impossible.

Proposition 3. Let $A, B$ be two A-IFSs determined by $\chi^{A}=\left(\mu_{A}, \nu_{A}\right), \chi^{B}=$ $\left(\mu_{B}, \nu_{B}\right) \in L^{X}$. If $\operatorname{Inf}_{x \in X} \nu_{A}(x)>0$ and $\operatorname{Inf}_{x \in X} \nu_{B}(x)>0$, then $A$ and $B$ are contradictory.

Proof. As $\operatorname{Inf}_{x \in X} \nu_{A}(x)>0,1-\operatorname{Inf}_{x \in X} \nu_{A}(x)<1$, and $\operatorname{Sup}_{x \in X}\left(1-\nu_{A}(x)\right)<1$, and so, there exists an $m<1$ such that $1-\nu_{A}(x) \leq m$ is satisfied for all $x \in X$; furthermore, 
$\mu_{A}(x) \leq 1-\nu_{A}(x) \leq m$ for all $x \in X$.

In the same way, as $\operatorname{Inf}_{x \in X} \nu_{B}(x)>0$, there exists an $n<1$ such that $\mu_{B}(x) \leq$ $1-\nu_{B}(x) \leq n$ for all $x \in X$.

As $n, m<1$, there exists an automorphism $g$ on $[0,1]$ such that $g(m)<1 / 2$ and $g(n)<1 / 2$. Then, for all $x \in X, g\left(\mu_{A}(x)\right)+g\left(1-\nu_{B}(x)\right) \leq g(m)+g(n)<1$ and $g\left(\mu_{B}(x)\right)+g\left(1-\nu_{A}(x)\right) \leq g(n)+g(m)<1$. Thus, $A$ and $B$ are $\mathcal{N}_{g}-$ contradictory, and therefore they are contradictory.

Proposition 4. Let $A, B$ be two A-IFSs determined by $\chi^{A}=\left(\mu_{A}, \nu_{A}\right), \chi^{B}=$ $\left(\mu_{B}, \nu_{B}\right) \in L^{X}$. If $A$ and $B$ are contradictory, then for all $x \in X$

$$
\operatorname{Sup}_{x \in X}\left(\mu_{A}(x)-\nu_{B}(x)\right)<1 \text { and } \operatorname{Sup}_{x \in X}\left(\mu_{B}(x)-\nu_{A}(x)\right)<1 .
$$

Proof. As $A, B$ are contradictory, they are $\mathcal{N}$-contradictory for some strong IFN $\mathcal{N}$. Let $\alpha_{N}$ be the equilibrium point of the fuzzy negation $N$ associated with $\mathcal{N}$. For each $x \in X$ such that $\mu_{A}(x) \leq \alpha_{N}$, obviously $\mu_{A}(x)-\nu_{A}(x) \leq \alpha_{N}$ holds. For each $x \in X$ such that $\mu_{A}(x)>\alpha_{N}$ then $N\left(\mu_{A}(x)\right)<N\left(\alpha_{N}\right)=\alpha_{N}$, thus $\mu_{A}(x)-\nu_{B}(x) \leq \mu_{A}(x)-1+N\left(\mu_{A}(x)\right)<\alpha_{N}$ since $N\left(\mu_{A}(x)\right)+\nu_{B}(x) \geq 1$. Therefore, $\operatorname{Sup}_{x \in X}\left(\mu_{A}(x)-\nu_{B}(x)\right) \leq \alpha_{N}<1$.

In a similar way, $\operatorname{Sup}_{x \in X}\left(\mu_{B}(x)-\nu_{A}(x)\right)<1$.

These results suggest us to give some functions in order to measure the contradictoriness between two A-IFSs. In particular, the following measures $\mathcal{C}_{1}$ and $\mathcal{C}_{2}$ are obtained from propositions 3 and 4 , respectively.

Definition 3. Let $A, B$ be two A-IFSs determined by $\chi^{A}=\left(\mu_{A}, \nu_{A}\right), \chi^{B}=$ $\left(\mu_{B}, \nu_{B}\right) \in L^{X}$. Then we define the $\mathcal{C}_{i}$-contradiction degree between $A$ and $B$, for $i=1,2$, as follows:

(i) $\mathcal{C}_{1}(A, B)=\operatorname{Min}\left(\operatorname{Inf}_{x \in X} \nu_{A}(x), \operatorname{Inf}_{x \in X} \nu_{B}(x)\right)$.

(ii) $\mathcal{C}_{2}(A, B)=0$ if $\operatorname{Inf}_{x \in X} \nu_{A}(x)=0$ or $\operatorname{Inf}_{x \in X} \nu_{B}(x)=0$,

$$
\mathcal{C}_{2}(A, B)=\operatorname{Min}\left(\operatorname{Inf}_{x \in X} \frac{1-\mu_{A}(x)+\nu_{B}(x)}{2}, \operatorname{Inf}_{x \in X} \frac{1-\mu_{B}(x)+\nu_{A}(x)}{2}\right) \text { in other case. }
$$

Remark 2. Let us note that for all $A, B \in \mathcal{A} \mathcal{I} \mathcal{F}(X)$ the above measures could be rewritten as

$$
\mathcal{C}_{1}(A, B)=\frac{\operatorname{Min}\left(d\left(\chi^{A B}(X), \mathcal{L}_{1}\right), d\left(\chi^{B A}(X), \mathcal{L}_{1}\right)\right)}{d\left(0_{\mathbf{L}}, \mathcal{L}_{1}\right)}
$$

being $d$ the euclidean distance and $\mathcal{L}_{1}=\left\{\left(\alpha_{1}, \alpha_{2}\right) \in[0,1]^{2}: \alpha_{2}=0\right\}$, and

$$
\mathcal{C}_{2}(A, B)=\frac{\operatorname{Min}\left(d_{1}\left(\chi^{A B}(X), \mathbf{1}_{\mathbf{L}}\right), d_{1}\left(\chi^{B A}(X), \mathbf{1}_{\mathbf{L}}\right)\right)}{d_{1}\left(\mathbf{0}_{\mathbf{L}}, \mathbf{1}_{\mathbf{L}}\right)}
$$


provided $\operatorname{Inf}_{x \in X} \nu_{A}(x)>0$ and $\operatorname{Inf}_{x \in X} \nu_{B}(x)>0$, and being $d_{1}$ the reticular (or Hamming) distance.

With a similar meaning, we propose a third measure:

Definition 4 . Let $A, B$ be two A-IFSs determined by $\chi^{A}=\left(\mu_{A}, \nu_{A}\right), \chi^{B}=$ $\left(\mu_{B}, \nu_{B}\right) \in L^{X}$. Then, we define the $\mathcal{C}_{3}$-contradiction degree between $A$ and $B$ as

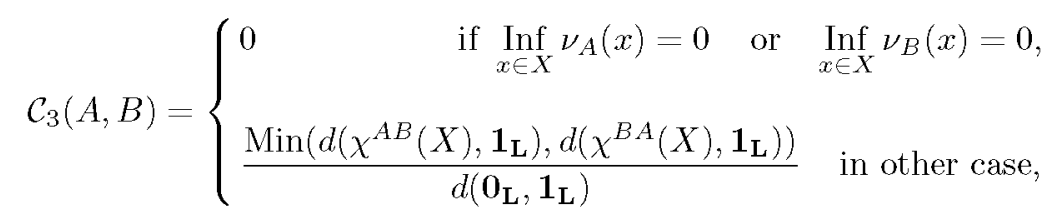

being $d$ the Euclidean distance.

Again, some properties are attained:

Theorem 2. For $i=1,2,3$, the function $\mathcal{C}_{i}: \mathcal{A I F}(X) \times \mathcal{A} \mathcal{I} \mathcal{F}(X) \rightarrow[0,1]$ given for each $A, B \in \mathcal{A T} \mathcal{T}(X)$ as in definitions 3 and 4 satisfies:

(i) $C_{i}$ is a symmetrical function: $\mathcal{C}_{i}(A, B)=\mathcal{C}_{i}(B, A)$ holds for all $A, B \in$ $\mathcal{A} \mathcal{I} \mathcal{F}(X)$.

(ii) $\mathcal{C}_{i}\left(0_{\mathrm{L}}, 0_{\mathrm{L}}\right)=1$.

(iii) If $\operatorname{Inf}_{x \in X} \nu_{A}(x)=0$ or $\operatorname{Inf}_{x \in X} \nu_{B}(x)=0$ then $\mathcal{C}_{i}(A, B)=0$.

(iv) $\mathcal{C}_{i}$ is anti-monotonic at both variables with respect to the orders $\leq_{L}$ in $L$ and the usual one in $\mathbb{R}$ : If $A, B, C \in \mathcal{A I \mathcal { F }}(X)$ with $\chi^{A} \leq_{L} \chi^{B}$ then $\mathcal{C}_{i}(B, C) \leq$ $\mathcal{C}_{i}(A, C)$.

Proof. Properties (i), (ii) and (iii) are immediate from definitions 3 and 4. For property $(i v)$, as $\nu_{B}(x) \leq \nu_{A}(x)$ for all $x \in X$, it is $\mathcal{C}_{1}(B, C) \leq \mathcal{C}_{1}(A, C)$. Besides, $1-\mu_{B}(x) \leq 1-\mu_{A}(x)$ and then $\left(1-\mu_{B}(x)\right)+\nu_{C}(x) \leq\left(1-\mu_{A}(x)\right)+\nu_{C}(x)$, and also $\left(1-\mu_{C}(x)\right)+\nu_{B}(x) \leq\left(1-\mu_{C}(x)\right)+\nu_{A}(x)$. Then, $\mathcal{C}_{2}(B, C) \leq \mathcal{C}_{2}(A, C)$. Finally,

$$
\begin{aligned}
d\left(\chi^{B C}(X), \mathbf{1}_{\mathbf{L}}\right) & =\operatorname{Inf}_{x \in X} \sqrt{\left(1-\mu_{B}(x)\right)^{2}+\nu_{C}(x)^{2}} \\
& \leq \operatorname{Inf}_{x \in X} \sqrt{\left(1-\mu_{A}(x)\right)^{2}+\nu_{C}(x)^{2}} \\
& =d\left(\chi^{A C}(X), \mathbf{1}_{\mathbf{L}}\right) \\
d\left(\chi^{C B}(X), \mathbf{1}_{\mathbf{L}}\right) & =\operatorname{Inf}_{x \in X} \sqrt{\left(1-\mu_{C}(x)\right)^{2}+\nu_{B}(x)^{2}} \\
& \leq \operatorname{Inf}_{x \in X} \sqrt{\left(1-\mu_{C}(x)\right)^{2}+\nu_{A}(x)^{2}} \\
& =d\left(\chi^{C A}(X), \mathbf{1}_{\mathbf{L}}\right)
\end{aligned}
$$


Therefore $\mathcal{C}_{3}(B, C) \leq \mathcal{C}_{3}(A, C)$.

Besides, we have the following relation between the measures $\mathcal{C}_{1}, \mathcal{C}_{2}$ and $\mathcal{C}_{3}$ defined in 3 and 4.

Proposition 5. For all $A, B \in \mathcal{A T \mathcal { F }}(X)$ the following inequalities hold,

$$
\mathcal{C}_{1}(A, B) \leq \mathcal{C}_{2}(A, B) \leq \mathcal{C}_{3}(A, B)
$$

Proof. As $\mathcal{C}_{1}(A, B) \leq \nu_{A}(x), \mathcal{C}_{1}(A, B) \leq \nu_{B}(x), \nu_{A}(x)+\nu_{B}(x) \leq \nu_{A}(x)+(1-$ $\left.\mu_{B}(x)\right)$ and $\nu_{A}(x)+\nu_{B}(x) \leq \nu_{B}(x)+\left(1-\mu_{A}(x)\right)$ for all $x \in X$, it is $\mathcal{C}_{1}(A, B) \leq$ $\frac{\nu_{A}(x)+\left(1-\mu_{B}(x)\right)}{2}$ and $\mathcal{C}_{1}(A, B) \leq \frac{\nu_{B}(x)+\left(1-\mu_{A}(x)\right)}{2}$ for all $x \in X$. Therefore $\mathcal{C}_{1}(A, B) \leq$ $\operatorname{Min}\left(\operatorname{Inf}_{x \in X} \frac{1-\mu_{A}(x)+\nu_{B}(x)}{2}, \operatorname{Inf}_{x \in X} \frac{1-\mu_{B}(x)+\nu_{A}(x)}{2}\right)=\mathcal{C}_{2}(A, B)$.

Now, as for all $x \in X$ it is

$$
\begin{aligned}
& \frac{\nu_{A}(x)+\left(1-\mu_{B}(x)\right)}{2} \leq \frac{\sqrt{\nu_{A}(x)^{2}+\left(1-\nu_{B}(x)\right)^{2}}}{\sqrt{2}} \\
& \frac{\nu_{B}(x)+\left(1-\mu_{A}(x)\right)}{2} \leq \frac{\sqrt{\nu_{B}(x)^{2}+\left(1-\mu_{A}(x)\right)^{2}}}{\sqrt{2}}
\end{aligned}
$$

then

$$
\begin{aligned}
\mathcal{C}_{2}(A, B) & \leq \operatorname{Min}\left(\operatorname{Inf}_{x \in X} \frac{\sqrt{\nu_{A}(x)^{2}+\left(1-\mu_{B}(x)\right)^{2}}}{\sqrt{2}} \operatorname{Inf}_{x \in X} \frac{\sqrt{\nu_{B}(x)^{2}+\left(1-\mu_{A}(x)\right)^{2}}}{\sqrt{2}}\right) \\
& =\mathcal{C}_{3}(A, B)
\end{aligned}
$$

\section{Self-Contradiction and Contradiction between two A-IFSs}

As it was remembered in the first section, the study of self-contradiction of an A-IFS was initiated in ${ }^{7}$. An A-IFS $A \in \mathcal{A} \mathcal{I F}(X)$ is said to be a self- $\mathcal{N}$-contradictory set with respect to any strong IFN, $\mathcal{N}$, if $\chi^{A}(x) \leq{ }_{L}\left(\mathcal{N} \circ \chi^{A}\right)(x)$ for all $x \in X$, where $\chi^{A}$ is the $L$-membership function of $A$. Besides, an A-IFS $A \in \mathcal{A} \mathcal{I F}(X)$ is said to be a self-contradictory set if $A$ is self- $\mathcal{N}$-contradictory regarding some strong IFN $\mathcal{N}$.

In general, there is not direct relation between the self-contradiction of two A-IFSs and the contradiction between them. In fact, let us see some examples.

Example 4. Let $A, B \in \mathcal{A} \mathcal{I} \mathcal{F}(X)$ be two A-IFSs on a non-empty set $X$ such that $\mu_{A}(x)=\nu_{A}(x)=1 / 2$ and $\mu_{B}(x)=\nu_{B}(x)=1 / 4$ for all $x \in X$. Then $\mu_{A}(x) \leq \nu_{A}(x)$ and $\mu_{B}(x) \leq \nu_{B}(x)$ for all $x \in X$ hold, and it implies that both $A$ and $B$ are self- $\mathcal{N}_{s}$-contradictory where $\mathcal{N}_{s}$ is the standard IFN (see ${ }^{5}$ ).

Nevertheless, $\mu_{A}(x)=1 / 2 \not \nu_{B}(x)=1 / 4$ for any $x \in X$, and then $A$ and $B$ are $\operatorname{not} \mathcal{N}_{s}$-contradictory.

Example 5. Let us consider the set $X=\{x, y\}$ and $A, B \in \mathcal{A I F}(X)$ given by $\chi^{A}(x)=(1 / 2,1 / 4), \chi^{A}(y)=(1 / 4,1 / 2), \chi^{B}(x)=(1 / 4,1 / 2)$ and $\chi^{B}(y)=(1 / 2,1 / 4)$. 
Both $A$ and $B$ are not self- $\mathcal{N}_{s}$-contradictory, as $\mu_{A}(x) \not \nu_{A}(x)$ and $\mu_{B}(y) \leq \nu_{B}(y)$. Nevertheless, $\mu_{A}(x) \leq \nu_{B}(x), \mu_{A}(y) \leq \nu_{B}(y), \mu_{B}(x) \leq \nu_{A}(x)$ and $\mu_{B}(y) \leq \nu_{A}(y)$, thus $A$ and $B$ are $\mathcal{N}_{s}$-contradictory between them.

Although these examples show the general independence between the $\mathcal{N}_{s^{-}}$ contradiction between two A-IFSs and the self- $\mathcal{N}_{s}$-contradiction of each of them, some results can be obtained.

Proposition 6. Let A, B be two A-IFSs given by $\chi^{A}=\left(\mu_{A}, \nu_{A}\right), \chi^{B}=\left(\mu_{B}, \nu_{B}\right) \in$ $L^{X}$, respectively, where $\mu_{A}(x)=\mu_{B}(x)$ for all $x \in X$. Then, $A, B$ are $\mathcal{N}$ contradictory if and only if both $A$ and $B$ are self-N-contradictory.

Proof. $A, B$ are $\mathcal{N}$-contradictory if and only if $g\left(\mu_{A}(x)\right)+g\left(1-\nu_{B}(x)\right) \leq 1$ and $g\left(\mu_{B}(x)\right)+g\left(1-\nu_{A}(x)\right) \leq 1$, that is equivalent to $g\left(\mu_{B}(x)\right)+g\left(1-\nu_{B}(x)\right) \leq 1$ and $g\left(\mu_{A}(x)\right)+g\left(1-\nu_{A}(x)\right) \leq 1$; that is, both $A$ and $B$ are self- $\mathcal{N}$-contradictory.

In the same way:

Proposition 7. Let $A, B$ be two A-IFSs given by $\chi^{A}=\left(\mu_{A}, \nu_{A}\right), \chi^{B}=\left(\mu_{B}, \nu_{B}\right)$, respectively, where $\nu_{A}(x)=\nu_{B}(x)$ for all $x \in X$. Then $A, B$ are $\mathcal{N}$-contradictory between them if and only if both $A$ and $B$ are self-N-contradictory.

Finally, let us see that some properties satisfied for self- $\mathcal{N}$-contradiction and selfcontradiction can not be directly translated to the case of contradiction between two A-IFSs.

For example, if an A-IFS $A$, with membership function $\mu_{A} \in[0,1]^{X}$, is self- $\mathcal{N}$ contradictory, then $\mu_{A}(x) \leq \alpha_{N}$, for all $x \in X \quad$ where $\alpha_{N}=g^{-1}\left(\frac{1}{2}\right)$ is the equilibrium point of the fuzzy negation $N$ associated with $\mathcal{N}$ and $g$ is the generator of $N$. But, in general, if $A, B$ are $\mathcal{N}$-contradictory, it could be $\mu_{A}(x)>\alpha_{N}$ and $\mu_{B}(x)>\alpha_{N}$ for some $x \in X$, as the following example shows.

Example 6. Let us consider the set $X=\{x, y\}$ and $A, B \in \mathcal{A I F}(X)$ given by $\chi^{A}(x)=(1 / 4,3 / 4), \chi^{A}(y)=(3 / 4,1 / 4), \chi^{B}(x)=(3 / 4,1 / 4)$ and $\chi^{B}(y)=(1 / 4,3 / 4)$. With the standard negation $\mathcal{N}_{s}, A$ and $B$ are $\mathcal{N}_{s}$-contradictory between them. However $\mu_{A}(y)=3 / 4>1 / 2$ and $\mu_{B}(x)=3 / 4>1 / 2$.

In a similar way, if an A-IFS $A$ with membership function $\mu_{A}(x)$, is selfcontradictory then $\mu_{A}(x)<1$, for all $x \in X$ (see ${ }^{7}$ ). But again, if $A, B$ are contradictory, then it could be $\mu_{A}(x)=1$ and $\mu_{B}(y)=1$ for some $x, y \in X$ :

Example 7. Let us consider the set $X=\{x, y\}$ and $A, B \in \mathcal{A} \mathcal{I} \mathcal{F}(X)$ given by $\chi^{A}(x)=(1,0), \chi^{A}(y)=(0,1), \chi^{B}(x)=(0,1)$ and $\chi^{B}(y)=(1,0) . A$ and $B$ are contradictory between them. Nevertheless $\mu_{A}(x)=1$ and $\mu_{B}(y)=1$.

However, another properties satisfied in self-contradiction could be, from a point of view, translated to contradiction between two A-IFSs. For example, in ${ }^{7}$ it was 
showed that if $\nu_{A}(x) \geq 1-\alpha_{N}$ for all $x \in X$, then $A$ is self- $\mathcal{N}$-contradictory. In our case:

Proposition 8. Given $A, B \in \mathcal{A} \mathcal{I} \mathcal{F}(X)$ determined by $\chi^{A}=\left(\mu_{A}, \nu_{A}\right), \chi^{B}=$ $\left(\mu_{B}, \nu_{B}\right) \in L^{X}$ and given $\mathcal{N}$ a strong $I F N$, if $\nu_{A}(x) \geq 1-\alpha_{N}$ and $\nu_{B}(x) \geq 1-\alpha_{N}$ for all $x \in X$, then $A, B$ are $\mathcal{N}$-contradictory.

Proof. As $\nu_{A}(x) \geq 1-\alpha_{N}$ and $\nu_{B}(x) \geq 1-\alpha_{N}$ with $\alpha_{N}=g^{-1}\left(\frac{1}{2}\right)$ being $g$ the generator of the negation $N$ associated to $\mathcal{N}$, the inequalities $g\left(1-\nu_{A}(x)\right) \leq \frac{1}{2}$ and $g\left(1-\nu_{B}(x)\right) \leq \frac{1}{2}$ are satisfied. Besides, as $\mu_{A}(x)+\nu_{A}(x) \leq 1$ and $\mu_{B}(x)+\nu_{B}(x) \leq 1$ it is hold that $g\left(\mu_{A}(x)\right) \leq g\left(1-\nu_{A}(x)\right) \leq \frac{1}{2}$ and $g\left(\mu_{B}(x)\right) \leq g\left(1-\nu_{B}(x)\right) \leq \frac{1}{2}$ and so $g\left(\mu_{A}(x)\right)+g\left(1-\nu_{B}(x)\right) \leq 1$ and $g\left(\mu_{B}(x)\right)+g\left(1-\nu_{A}(x)\right) \leq 1$.

Also, in ${ }^{7}$ it was showed that if $\nu_{A}(x)>0$ for all $x \in X$, then $A$ is selfcontradictory. In our case, we have the result given in proposition 3: " $\operatorname{Inf}_{x \in X} \nu_{A}(x)>0$ and $\operatorname{Inf}_{x \in X} \nu_{B}(x)>0$, then $A, B$ are contradictory".

Besides in the same paper, it was obtained that if $\mu_{A}(x)>\alpha_{N}$ for all $x \in X$, then $A$ is not- $\mathcal{N}$-contradictory. Now, we have:

Proposition 9. Given $A, B \in \mathcal{A I F}(X)$ determined by $\chi^{A}=\left(\mu_{A}, \nu_{A}\right), \chi^{B}=$ $\left(\mu_{B}, \nu_{B}\right) \in L^{X}$ and given $\mathcal{N}$ a strong IFN, if $\mu_{A}(x)>\alpha_{N}$ and $\mu_{B}(x)>\alpha_{N}$ for all $x \in X$, then $A, B$ are not $\mathcal{N}$-contradictory.

Proof. As $\mu_{A}(x)>g^{-1}\left(\frac{1}{2}\right), \mu_{B}(x)>g^{-1}\left(\frac{1}{2}\right), 1-\nu_{A}(x) \geq \mu_{A}(x)$ and $1-\nu_{B}(x) \geq$ $\mu_{B}(x)$ for all $x \in X$, then $g\left(\mu_{A}(x)\right)+g\left(1-\nu_{B}(x)\right)>1$ and $g\left(\mu_{B}(x)\right)+g\left(1-\nu_{A}(x)\right)>$ 1 for all $x \in X$.

Remark 3. Last propositions point out that if $\chi^{A}(X)$ and $\chi^{B}(X)$ remain into the striped area (figure 6), which is included in the self- $\mathcal{N}$-contradiction region, then $A$, $B$ are $\mathcal{N}$-contradictory; and if $\chi^{A}(X)$ and $\chi^{B}(X)$ remain into the grid area (figure 6 ), which is included in the non-self- $\mathcal{N}$-contradiction region, then $A, B$ are not $\mathcal{N}$-contradictory.

\section{Contradiction degrees and Self-contradiction degrees}

As it was said in section 4, there is not direct relation between the self-contradiction of two A-IFSs and the contradiction between them. But, however, we can consider self-contradiction as a particular case from contradiction.

We remember that, given $A, B \in \mathcal{A} \mathcal{I} \mathcal{F}(X)$ determined by $\chi^{A}=\left(\mu_{A}, \nu_{A}\right), \chi^{B}=$ $\left(\mu_{B}, \nu_{B}\right) \in L^{X}$ and given $\mathcal{N}$ a strong IFN, $A$ and $B$ are $\mathcal{N}$-contradictory if $\chi^{A}(x) \leq{ }_{L}$ $\left(\mathcal{N} \circ \chi^{B}\right)(x)$, for all $x \in X$. And, $A$ is self- $\mathcal{N}$-contradictory if $\chi^{A}(x) \leq_{L}\left(\mathcal{N} \circ \chi^{A}\right)(x)$, for all $x \in X$. Therefore, the definition of self- $\mathcal{N}$-contradictory intuitionistic fuzzy set is a particular case from that of $\mathcal{N}$-contradictory intuitionistic fuzzy sets, where the two sets are the same. 


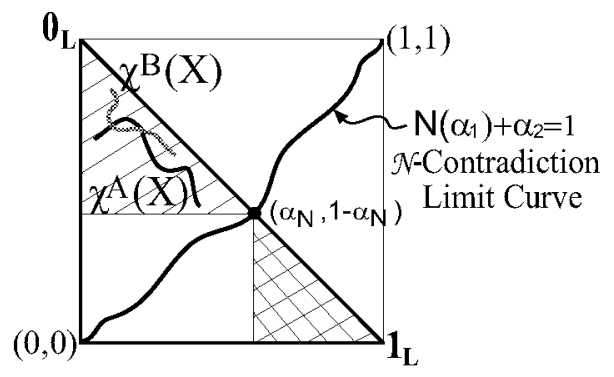

Fig. 6. Contradiction area and Non-Contradiction area.

In ${ }^{7}$ it was showed that if $\nu_{A}(x) \geq 1-g^{-1}(1 / 2)$, where $g$ is the generator of the negation $N$ associated to $\mathcal{N}$, then $A$ is self- $\mathcal{N}$-contradictory. And, if $A$ is self$\mathcal{N}$-contradictory then $\mu_{A}(x) \leq g^{-1}(1 / 2)$. Again, this property can be obtained as a particular case from lemma 1 .

Too, let us remember that, $A, B \in \mathcal{A} \mathcal{I} \mathcal{F}(X)$ are contradictory if they are $\mathcal{N}$ contradictory regarding some strong IFN $\mathcal{N}$; and $A$ is self-contradictory if $A$ is self- $\mathcal{N}$-contradictory regarding some strong IFN $\mathcal{N}$. Again, the definition of selfcontradictory intuitionistic fuzzy set is a particular case from that of contradictory intuitionistic fuzzy sets, where the two sets are the same.

In ${ }^{7}$ it was proved that if $\operatorname{Inf}_{x \in X} \nu_{A}(x)>0$, then $A$ is self-contradictory. Again, this property can be obtained as a particular case from proposition 3 .

On the other hand, the property "if $A$ is self-contradictory then $\operatorname{Sup}_{x \in X} \mu_{A}(x)<1$ ", can not be obtained as a particular case from some property for contradiction between two A-IFSs, since $A$ and $B$ contradictory does not imply neither $\operatorname{Sup} \mu_{A}(x)<$ 1 nor $\operatorname{Sup}_{x \in X} \mu_{B}(x)<1$, as it was showed in the example 7 .

Taking into account that self-contradiction could be view as contradiction of a set with itself, the degrees of contradiction defined in this paper, provide us the respective degrees of self-contradiction.

So, the $\mathcal{N}$-contradiction degrees given in definition 2 are turned into the following self- $\mathcal{N}$-contradiction degrees:

(i) $\mathcal{C}_{1}^{\mathcal{N}}(A)=\operatorname{Max}\left(0, \operatorname{Inf}_{x \in X}\left(N_{g}\left(\mu_{A}(x)\right)+\nu_{A}(x)-1\right)\right)$

(ii) $\mathcal{C}_{2}^{\mathcal{N}}(A)=\operatorname{Max}\left(0,1-\operatorname{Sup}_{x \in X}\left(g\left(\mu_{A}(x)\right)+g\left(1-\nu_{A}(x)\right)\right)\right)$

(iii) $\mathcal{C}_{3}^{\mathcal{N}}(A)=\frac{d\left(\chi^{A A}, \mathcal{R}_{\mathcal{N}}^{*}\right)}{d\left(\mathbf{0}_{\mathbf{L}}, \mathcal{R}_{\mathcal{N}}^{*}\right)}=\frac{d\left(\chi^{A}, \mathcal{L}_{\mathcal{N}}^{*}\right)}{d\left(\mathbf{0}_{\mathbf{L}}, \mathcal{L}_{\mathcal{N}}^{*}\right)}$

being $\mathcal{L}_{\mathcal{N}}^{*}=\left\{\left(\alpha_{1}, \alpha_{2}\right) \in L ; N\left(\alpha_{1}\right)+\alpha_{2} \leq 1\right\}$, and taking into account that $d\left(\mathbf{0}_{\mathrm{L}}, \mathcal{R}_{\mathcal{N}}^{*}\right)=d\left(\mathbf{0}_{\mathrm{L}}, \mathcal{L}_{\mathcal{N}}^{*}\right)$ since the $\mathcal{N}$-boundary curve is symmetrical. 
These measures of self- $\mathcal{N}$-contradiction coincide with the measures of self- $\mathcal{N}$ contradiction defined

In the same way, the contradiction degrees given in definitions 3 and 4 are turned into the following self-contradiction degrees:

(i) $\mathcal{C}_{1}(A)=\operatorname{Inf}_{x \in X} \nu_{A}(x)=\frac{d\left(\chi^{A}, \mathcal{L}_{1}\right)}{d\left(0_{\mathrm{L}}, \mathcal{L}_{1}\right)}$

(ii) $\mathcal{C}_{2}(A)=\left\{\begin{array}{l}0 \quad \text { if } \operatorname{Inf}_{x \in X} \nu_{A}(x)=0 \\ \operatorname{Inf}_{x \in X} \frac{1-\mu_{A}(x)+\nu_{A}(x)}{2}=\frac{d_{1}\left(\chi^{A}, \mathbf{1}_{\mathbf{L}}\right)}{d_{1}\left(\mathbf{0}_{\mathrm{L}}, \mathbf{1}_{\mathrm{L}}\right)} \quad \text { in other case }\end{array}\right.$

(iii) $\mathcal{C}_{3}(A)= \begin{cases}0 & \text { if } \operatorname{Inf}_{x \in X} \nu_{A}(x)=0, \\ \frac{d\left(\chi^{A}, \mathbf{1}_{\mathbf{L}}\right)}{d\left(\mathbf{0}_{\mathbf{L}}, \mathbf{1}_{\mathbf{L}}\right)} & \text { in other case }\end{cases}$

Again, these measures of self-contradiction coincide with the measures of selfcontradiction defined in ${ }^{5}$.

\section{Conclusions}

Some functions to measure the contradiction between two intuitionistic (or Atanassov's) fuzzy sets have been proposed, both depending on a given intuitionistic negation, and independently on any specific negation. The basic properties of these functions have been shown. Furthermore, some results about the relation between self-contradiction, of each one of two intuitionistic fuzzy sets, and the contradiction between both of them, have been given.

\section{References}

K. T. Atanassov, Intuitionistic fuzzy sets (Physica-Verlag, Heidelberg, New York, 1999). H. Bustince, J. Kacprzyk and V. Mohedano, "Intuitionistic fuzzy generators - Application to intuitionistic fuzzy complementation", Fuzzy Sets and Systems 114 (2000) $485-504$.

E. Castiñeira, S. Cubillo and S. Bellido, "Degrees of Contradiction in Fuzzy Sets Theory", Proceedings IPMU'02, Annecy (France), 2002, pp. 171-176.

E. Castiñeira, S. Cubillo and S. Bellido, "Contradicción entre dos conjuntos", Actas ESTYLF'02, León (Spain), 2002, pp. 379-383 (in Spanish).

E. Castiñeira, S. Cubillo, C. Torres and V. Zarzosa, "Selft-Contradiction Degrees in Intuitionistic Fuzzy Sets", Proceedings of Joint EUSFLAT-LFA 2005, Barcelona (Spain), 2005, pp. 455-460.

E. Castiñeira, S. Cubillo and C. Torres, "Searching Degrees of Selft-Contradiction in Atanassov's Fuzzy Sets", Mathware and Soft Computing (2006) (accepted).

S. Cubillo and E. Castiñeira, "Contradiction in Intuitionistic Fuzzy Sets", Proceedings IPMU'04, Perugia (Italy), 2004, pp. 2180-2186.

G. Deschrijver, C. Cornelis and E. Kerre, "Intuitionistic fuzzy connectives revisited", Proceedings IPMU'02, Annecy (France), 2002, pp. 1839-1844. 
J. A. Goguen, "L-Fuzzy Sets", Journal of Mathematical Analysis and Applications 18(1) (1967) 623-668.

E. Trillas, "Sobre funciones de negación en la teoría de conjuntos difusos", Stochastica III/1 (1979) 47-60 (in Spanish). Reprinted (English version) (1998) in Avances of Fuzzy Logic (eds. S. Barro et altri), 31-43 (Ed. Universidad de Santiago de Compostela).

E. Trillas, C. Alsina and J. Jacas, "On Contradiction in Fuzzy Logic", Soft Computing 3(4) (1999) 197-199.

E. Trillas and S. Cubillo, "On Non-Contradictory Input/Output Couples in Zadeh's CRI", Proceedings NAFIPS, New York, 1999, pp. 28-32. 